Article

\title{
Grant-Free Resource Allocation for NOMA V2X Uplink Systems Using a Genetic Algorithm Approach
}

\author{
Sol Lee ${ }^{\circledR}$, Jeehyeong Kim $₫$, Joohan Park $₫$ and Sunghyun Cho* $*$ \\ Department of Computer Science, Hanyang University, Gyeonggi-do 15588, Korea; \\ dlthfhy@hanyang.ac.kr (S.L.); manje111@hanyang.ac.kr (J.K.); 1994pjh@hanyang.ac.kr (J.P.) \\ * Correspondence: chopro@hanyang.ac.kr
}

Received: 3 June 2020; Accepted: 5 July 2020; Published: 8 July 2020

\begin{abstract}
While NOMA-V2V (non-orthogonal multiple accesscan-vehicle-to-vehicle) effectively achieve massive connectivity requirements in 5G network systems, minimizing communication latency is a very crucial challenge. To address the latency problem, we propose a channel allocation method called hyper-fraction, which divides the road into many zones and allocates a channel to each zone. Then, a vehicle located within the corresponding zone uses the channel allocated to the zone. Hyper-fraction will allow the system to minimize communication latency between a user equipment (UE) and a base station (BS) caused by scheduling processes and consequentially reduce the overall latency of the system. In the simulation, a novel concept of genetic algorithm (GA) is utilized, called GA with continuous pool. It is an approach to enable conventional GA to solve optimization problems for continuous situations within much less computation, especially in situations where the elements in the system keep moving such as vehicular networks. As a result, GA with continuous pool is proven to be an effective heuristic method to improve throughput rate, as well as hyper-fraction improving the latency of NOMA V2V and vehicle-to-infrastructure (V2I) systems.
\end{abstract}

Keywords: NOMA; V2V; V2I; resource allocation; hyper-fraction; genetic algorithm; continuous pool

\section{Introduction}

With the explosive growth in the number of wireless users and data demands, the current generation wireless network will not be able to keep up with the demands. To solve the problem, the next-generation $5 \mathrm{G}$ network is introduced and it focuses on providing users with very high-data-rate, low-latency, and high-capacity wireless services. Since the network will become denser over time [1], several solutions to address the ever-increasing needs have been studied and included in the 5G network [2]. In particular, mobility situations such as vehicular networks are considered very important challenges of 5G network to provide satisfactory service to users traveling at high speed [3,4]. In mobility situations, the channel state information (CSI) keeps changing because the locations of devices vary over time as they move, making the optimization problem very difficult [5].

Vehicle-to-everything (V2X) communication is the most representative and important example of mobility situations. As intelligent transportation systems are being broadly implemented, communications between vehicles, networks, and infrastructures are considered to be very crucial parts in terms of road safety and effective transport-related services [6].

However, to meet the various requirements of V2X communications including safety and efficiency that are mentioned above, high system throughput and low latency need to be achieved [7]. To satisfy such criteria, one of the newer additions to multiple access schemes, non-orthogonal multiple access (NOMA), is perceived as a very promising candidate [8].

NOMA is initially proposed as an improved multiple access scheme to maximize the utilization of radio resources [9-11] since the number of users in the future wireless systems will dramatically 
increase [12]. NOMA is considered one of the key technologies in the 5G network to meet the requirements such as massive connectivity $[13,14]$. NOMA improves system throughput by allowing multiple users to share the same time and frequency resources, which effectively alleviates the massive connectivity problem and therefore NOMA is widely researched for the purpose of connectivity maximization in wireless systems $[15,16]$.

Since NOMA is effective in achieving high system throughput, it can be used to improve the throughput rate in vehicular networks where resources such as time are scarce due to the nature of high-speed devices and also because the number of vehicles adopting V2X communications will keep increasing.

In V2X, the latency requirements are especially strict since it is closely related to road safety [17]. It means that users must effectively use their limited resources within a short time because delayed communications can lead to traffic congestion or car accidents. In addition, increased usage of smaller base stations (BS) such as macrocells and femtocells for V2I communication narrows down the coverage area per BS compared to bigger BSs and therefore reduces the time available for using a base station, meaning that it is very important to efficiently use the resources within a limited time [18].

Therefore, introducing NOMA to V2X significantly improves the quality of service of the system with respect to system throughput $[19,20]$. However, resource allocation in NOMA is a difficult challenge and it can even cause more delays and lower the throughput in the system if poorly conducted. This is because resource allocation in NOMA is generally more complex than in OMA schemes. In addition, uplink in NOMA V2X is a difficult problem because the system contains vehicles, which keep moving therefore making it harder to allocate resources correctly and fairly in its ever-changing characteristics.

To overcome both the latency and user throughput problem in NOMA systems, we propose two novel methods for grant-free resource allocation called hyper-fraction and genetic algorithm (GA) with continuous pool. Using hyper-fraction, the system can provide low latency between BSs and user equipments (UE). In addition, using GA with a continuous pool, the system can quickly achieve high system throughput and provide fairness among users in vehicular systems. Compared to using fractional frequency reuse (FFR) [21], hyper-fraction can allocate the radio resources to users within minimum latency because a BS does not need to communicate with a UE for additional scheduling. Instead, a UE only needs to know its own location to choose which channel to use and also which BS to communicate with, enabling much lower-latency communication which is crucial for future radio communications. In addition, compared to using conventional GA [22], GA with continuous pool can solve optimization problems in continuous situations much faster and effectively because it uses the previously converged solution and keeps improving it even further. Our contributions in this paper are as follows:

- We provide a novel concept called hyper-fraction, which minimizes the latency between BS and UE for the resource allocation scheduling process.

- We provide a novel approach of using continuous pool in GA and prove that it is suitable for solving the resource allocation problem in vehicular situations.

- We present an extended concept of the mutation procedure of GA which is effective for channel allocation problems in NOMA device-to-device (D2D) systems.

This paper is organized as follows: In Section 2, we review the related works about NOMA, GA-based resource allocation schemes and FFR. In Section 3, we elaborate on the concept of hyper-fraction and the advantages of using it. In addition, we describe the system model and the simulation, and then formulate the problem of resource allocation in NOMA V2V and V2I uplink systems and the proposed scheme. In Section 4, we explain the basic workflow of GA and the adoption of continuous pool in GA, then the following advantages of using continuous pool in resource allocation for vehicular networks. In Section 5, the results of the simulation are given. In Section 6, we discuss the results and potential future works to proceed. 


\section{Related Works}

\subsection{NOMA}

NOMA is considered a very promising technology as a future radio multiple access scheme. It is superior to current orthogonal multiple access (OMA) schemes in terms of system throughput and connectivity, satisfying the high data rate and massive connection density requirements in $5 \mathrm{G}$ systems [23]. In NOMA, the user on the transmit side sends one superposed signal consisting of usually two different signals within the same time and frequency slot to the users on the receive side. Two different signals within one superposed signal can be decoded by the receiver users because different power levels are assigned to each user. More specifically, the NOMA-near user, who is in a better channel condition performs successive interference cancellation (SIC) to subtract the stronger signal sent for the NOMA-far user and then decodes the signal. Then, the NOMA-far user, who is in a worse channel condition will decode the signal without a further process because the signal for the NOMA-near user would have become very weak by the time it arrives that it can be treated as noise.

Although early NOMA studies mainly consider downlink communication systems, the NOMA uplink system is also considered and analyzed in the field as an important issue to improve connectivity and maximize system throughput of uplink transmissions [24-26]. Furthermore, as vehicular communications emerge as one of the core prerequisites for safe autonomous driving and road traffic control, vehicle-to-vehicle (V2V) and vehicle-to-infrastructure (V2I) communications need to meet the low-latency and high-reliability requirements. To address such problems, several studies have introduced NOMA in V2X communications and verified that NOMA is effective in V2X communications for high system throughput and low-latency [27]. In addition, several studies address system analysis and resource allocation problems in the NOMA V2X system [28,29].

However, previous studies on NOMA V2X systems that focus on resource allocation and latency problems still require constant scheduling processes which inevitably cause delays. Therefore, a novel approach to channel allocation which can significantly reduce the latency is necessary for minimized latency.

\subsection{GA-Based Resource Allocation}

For UE in NOMA systems to have as maximized throughput rate as possible, resource allocation methods play a very important role in improving system throughput. In order to solve the resource allocation problem, studies have been conducted using optimization algorithms. One of the studies addressing the resource allocation problem proposes particle swarm optimization to maximize signal-to-interference-plus-noise ratio (SINR) and increase total user capacity [30]. Particle swarm optimization is one of the evolutionary computation methodologies that is used to find the approximate optimal solutions for non-convex problems using the idea of inertia and acceleration which are constantly adjusted by measuring how far current values are to the best solution.

Similar to particle swarm optimization, GA is also used as a relatively faster and computationally less-complex search heuristic for solving non-convex problems. GA adopts the idea of natural selection and survival of the fittest. GA performs selection, crossover, and mutation based on fitness function that scores how fit each individual is in terms of solving the given optimization problem. To address the resource allocation problem in NOMA systems, a study uses GA to maximize system throughput and improve user fairness [22]. More specifically, GA is used to determine optimal transmission power for users and also to group users properly in respect of maximizing user throughput. GA is proven to be effective in achieving the optimal trade-off between system throughput and user fairness by maximizing the geometric mean of the user throughput. At the same time, GA converges to the same final result as exhaustive search, which iterates through every possible solution, within much fewer iterations. As previous studies demonstrate, evolutionary algorithm-based methods such as GA are proven to be an effective and powerful heuristic method with lower computational complexity and relatively quick convergence. 
Despite the fact that GA methods can improve the system throughput in resource allocation, it is not easy to apply conventional GA methods in continuous situations because every time the channel state changes, the algorithm will have to find the optimal solution all over again to reflect the changes, which leads to more time and computational complexity.

\subsection{Fractional Frequency Reuse}

While NOMA performs a great job in improving system throughput and connectivity, there is still a user fairness problem where users near the edge of the cell coverage have a low throughput rate due to the degraded signals from additional interference. Therefore, some studies approached the user fairness problems in NOMA by using the FFR scheme in NOMA systems [21]. FFR utilizes the unused parts of resource blocks and allocates them to users served by different BS within the same tier, maximizing the throughput rate of users and improving the fairness among the users both in uplink systems [31], and device-to-device (D2D) systems [32].

However, in order to use FFR, additional communications of UEs and BSs for exchanging scheduling information are required, which can be a drawback for situations where low latency is very important.

\section{System Model}

\subsection{Hyper-Fraction}

Hyper-fraction is originally inspired by FFR, which divides the cell coverage area into geographical sectors and enables the reuse of radio resources with scheduling and allocation. In hyper-fraction, the system also allocates channels to UEs based on their geographical locations. However, hyper-fraction does not rely on the scheduling process but instead it instantly allocates channels to UEs based on their geographical locations. In addition, hyper-fraction divides the area more specifically and also much smaller. To be more specific, the system divides the BS coverage area, which, in our case, the road into many hyper-fraction zones, namely HF-zones, which are square-shaped areas with the same size each as shown in Figure 1. The system allocates a different channel to each HF-zone and a UE located within the corresponding HF-zone will use the channel which is allocated to that zone.

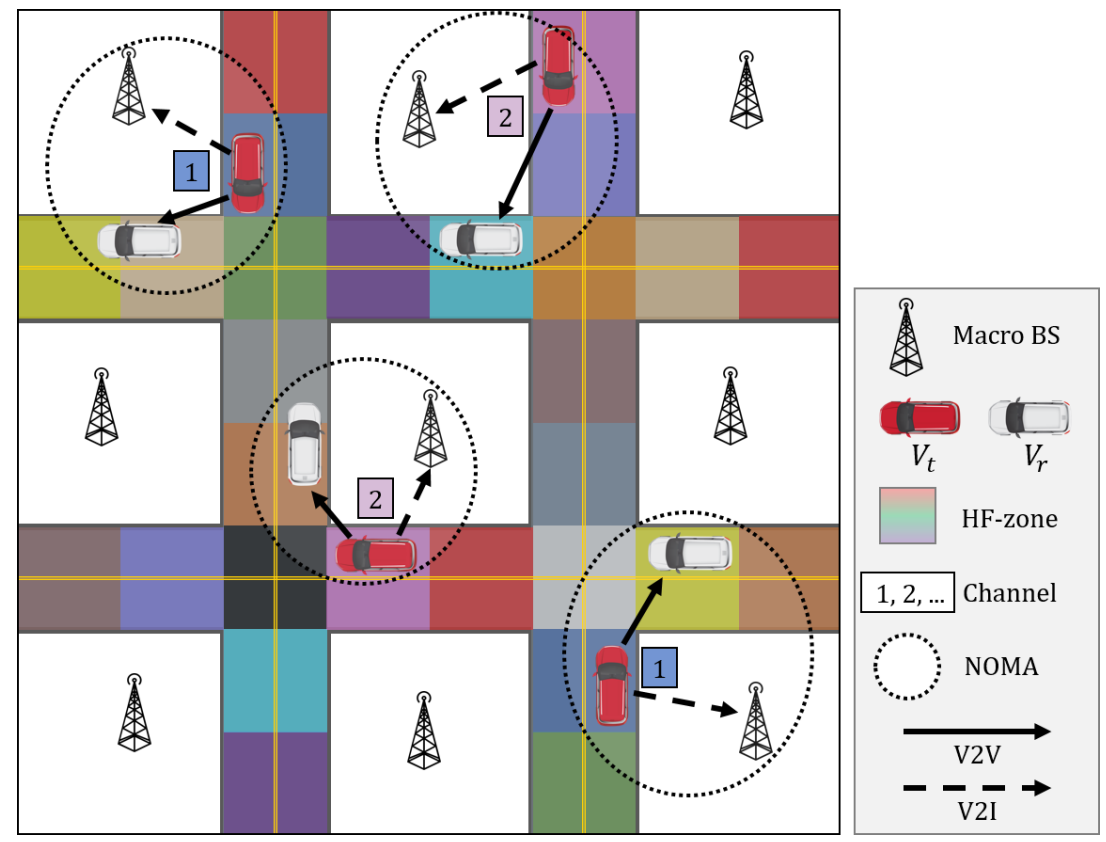

Figure 1. A representation of the simulation. HF-zones (hyper-fraction zones) with the same color share the same channel. Therefore, vehicles on the HF-zones with the same color share the same channel. 
The channel data for HF-zones are stored in a resource allocation (RA) table. The RA table is an array that contains channel numbers assigned to each HF-zone. Once the UE successfully collects the RA table, the UE does not need any additional information or scheduling process for channel allocation. However, it is still required that the system knows which channel should be assigned to which HF-zone for maximum system throughput rate. To solve that, we use GA with a continuous pool.

\subsection{Target System}

We assume a system based on the Manhattan mobility model, where $k$ vehicles are randomly placed following the Gaussian distribution and moving along the road simultaneously with the difference speeds. Specifically, each one-third of the vehicles move at $36 \mathrm{~km} / \mathrm{h}, 72 \mathrm{~km} / \mathrm{h}$, and $108 \mathrm{~km} / \mathrm{h}$, respectively. More specifically, a vehicle $V_{t}$ transmits V2V and V2I signals using NOMA. A receiving vehicle on a V2V link is denoted as $V_{r}$ where $V_{t}, V_{r} \in \mathcal{V}$. In addition, $b$ macro base stations (MBS) that receive V2I signal from $V_{t}$ and $z$ hyper-fraction zones are in the system where $b \in \mathcal{B}$. The system model is shown and described in Figure 1.

In the target system, all vehicles perform V2V and V2I NOMA communication through the channel that is allocated to the hyper-fraction zone based on their geographical location. Each vehicle is connected to another vehicle and a BS. The proposed method will try to find the NOMA pairs with the highest total throughput rate possible.

The proposed method and the target system in this paper can be applied in areas such as vehicle platooning and co-operative driving to improve safety and efficiency of autonomous driving. For example, the vehicles should be connected to each other with high throughput rate to share enough information about themselves such as speed, direction, position, identity, and data collected by various sensors. In addition, vehicles need to communicate with a BS so that the BS can help them acquire more information about the road.

\subsection{Problem Formulation}

We propose a grant-free resource allocation method based on GA. To define channel model, we consider small scale fast fading, shadowing, and large scale path loss. According to [5], channel gain between a transmitter $i$ and a receiver $j$ over the channel $n \in \mathcal{N}$ is defined as

$$
H_{i, j}^{n}=\left|h_{i, j}^{n}\right|^{2} \beta_{i, j} C_{i, j} d_{i, j}^{-\alpha_{i}}
$$

where $\mathcal{N}=\{1,2, \ldots, N\}$. A transmitter $i$ is the $i$ th vehicle device $V_{i}$ in $\mathcal{V}$ as a transmitter device $V_{t} . j$ denotes two types of receiver devices $\{v, b\}$ because there are two types of receiver: a $v$ th $\mathrm{V} 2 \mathrm{~V}$ receiver $V_{r}$ and a $b$ th receiving base station. $\beta_{i, j}$ is a log-normal shadowing random variable with standard deviation $\zeta_{i, j}$ for the link between $i$ th transmitter and $j$ th receiver. $C_{i, j}$ is defined as a path-loss constant between $i$ th transmitter and $j$ th receiver. $d_{i, j}$ is the distance between $i$ th transmitter and $j$ th receiver. $\alpha_{i}$ is the path-loss exponent for $i$ th transmitter. $h_{i, j}^{n}$ is the fast fading component of the link between $i$ th transmitter and $j$ th receiver on the $n$th channel as a complex Gaussian distribution with zero mean and unit variance, where $h_{i, j}^{n} \sim \mathcal{C N}(0,1)$. The transmitter vehicle is always transmitting signals to both the receiver vehicle and the base station.

From the channel definition in Equation (1), we can derive signal-interference-noise-ratio (SINR) for V2V and V2I, respectively. To define SINR of V2V, we can define $v$ as a vth vehicle device that is determined as a receiver for the transmitter $i$. Then, SINR between $i$ th transmitter and $v$ th receiver, $\eta_{i, v}$, can be defined as:

$$
\eta_{i, v}^{n}=\frac{P_{i, v} H_{i, v}^{n}}{\sum_{s \in \mathcal{V}} a_{s}^{n}\left(P_{s, v}+P_{s, b}\right) H_{s, v}^{n}+\sigma^{2}}
$$

where $P_{i, v}$ is the transmit power for $\mathrm{V} 2 \mathrm{~V}$ link from $i$ th transmitter to $v$ th receiver and $P_{i, b}$ is the transmit power for V2I link between $i$ th transmitter and $b$ th receiving base station. Note that there is interference from $s$ because more than one data transmission can exist over the same channel occasionally in the 
proposed scheme. Because the interference source sth device sends V2V and V2I signals at the same time by using NOMA, the transmit power of the $s$ th device is $P_{s, v}+P_{s, b}$. In such situations, minimizing the effects of mutual interference is the key idea. To describe the interference sources, $a_{s}^{n}$ is an allocation indicator where $a_{s}^{n} \in\{0,1\}$. If the device $s$ is allocated to use the $n$th channel, $a_{s}^{n}$ is set to 1 . Otherwise, it is $0 . \sigma^{2}$ is the noise power. Interference from the V2I link of the same device is canceled according to successive interference cancellation (SIC) [12]. SINR of V2I link between $i$ th transmitter and $b$ th receiver can be defined as:

$$
\eta_{i, b}^{n}=\frac{P_{i, b} H_{i, b}^{n}}{\sum_{s \in \mathcal{V}} a_{s}^{n}\left(P_{s, v}+P_{s, b}\right) H_{s, b}^{n}+P_{i, v}^{n} H_{i, v}^{n}+\sigma^{2}}
$$

Thus, the throughput of V2V and V2I links for ith transmitting device can be formulated as:

$$
\begin{aligned}
& R_{i, v}=\sum_{n \in \mathcal{N}} a_{i}^{n} \log _{2}\left(1+\eta_{i, v}^{n}\right) \\
& R_{i, b}=\sum_{n \in \mathcal{N}} a_{i}^{n} \log _{2}\left(1+\eta_{i, b}^{n}\right)
\end{aligned}
$$

As a result, we can formulate the problem of maximizing the total throughput of the system as follows:

$$
\begin{array}{ll}
\text { maximize } & \sum_{n \in \mathcal{N}} \sum_{i \in \mathcal{V}} R_{i, v}^{n}+R_{i, b}^{n} \\
\text { subject to } & a_{i}^{n} \in\{0,1\}, \quad \forall n, i, \\
& \sum_{n \in \mathcal{N}} a_{i}^{n}=1, \quad \forall i, \\
& \frac{\sum_{n \in \mathcal{N}} a_{i}^{n}\left(P_{i, b}-P_{i, v}\right) H_{i, v}^{n}}{\sigma^{2}}>L_{0}, \quad \forall i
\end{array}
$$

where Equation (6b) defined the channel indicator. If $a_{i}^{n}$ is 1 , the $i$ th vehicle device using $n$th channel as a transmitter. At the same time, a V2I link between a corresponding $b$ th receiving base station and $i$ th transmitter over $n$th channel is also allocated. Equation (6c) implies that a device can use only one channel at the same time. Equation (6d) is a power difference condition for NOMA, as described in [33]. To use SIC in the vehicle receiver, the receiving signal power for V2I link has to be much stronger than that of the V2V link. There is a premise that the transmitter is much closer to the receiver than the macro base station $b$ so the signal power of a V2V link can be much less than that of a V2I link. In situations where this premise is not established, transmit power control will be excessively inefficient if the system uses NOMA. Therefore, we assume NOMA is not adopted in that case, which is a situation where the transmitter is closer than the receiver.

\section{The Proposed Genetic Algorithm with Continuous Pool}

Conventional GA consists of fitness function, selection, crossover, and mutation. First, it creates the initial population consisting of random individuals which are usually arrays or matrices. Then, GA scores each individual by measuring how much the individual is fit for solving the problem (fitness function) and decides which individuals have the most proper and fittest elements, based on the fitness score (selection). With the selected individuals, the algorithm usually combines two of them by randomly choosing some of the elements in an individual and exchange them with some elements in another individual (crossover). After performing crossover, a few elements of the individuals randomly change for more exploration of possible solutions (mutation). Finally, one generation is over and the population keeps evolving by iterating through generations until it converges to the optimal solution or until it reaches the final generation manually set by the system. 
In this paper, a method based on GA is used to improve the RA table in terms of maximizing the total throughput rate of the system. Therefore, an individual represents an RA table, an element represents the channel number in the RA table, and the population represents the group of all RA tables, which we also call 'the pool'.

Though our scheme keeps the core idea of GA, some modifications are made so that the algorithm can be more suitable for our specific problem. Two main differences between conventional GA and GA with continuous pool are firstly the continuity of individuals in the final generation and secondly the different procedures in the evolution process. In GA with continuous pool, when an episode is over as GA reaches the final generation, individuals in the last generation are passed on to the first generation of the next episode. As a result, the system will need much less computational time to converge in the following episodes. This is because our target system has moving vehicles whose movements are continuous, meaning that the locations of vehicles do not change in a great measure at once.

Specifically compared to conventional GA, the proposed method can achieve higher performance in systems where the channel state information, especially the locations of devices, tend to change gradually. It is possible because the proposed method keeps the resource allocation information which have converged in the last episode and uses it in the current episode. Since we assume in our system model that the devices do not travel a great distance in each episode, the proposed method can use the resource allocation information which it had used in the previous episode and further improve the resource allocation with the help of GA.

The proposed method can outperform conventional GA because of the following reason. After the devices have moved, the proposed method starts its evolution process from the previously converged resource allocation table in the last episode. Thus, it does not only have possibly the best resource allocation solution for the current situation without further computations, but it can also improve resource allocation in the current situation with a further evolution process. On the other hand, it takes much more computational time with a conventional GA approach when a new episode begins since it must start the evolution process over again from the beginning without any prior information about the last episode. Therefore, the proposed method can have more improved results as the system operates for more episodes because of its continuous characteristics.

Since the proposed method aims to solve the resource allocation problem, the evolution process of our proposed GA method is modified to perform resource allocation more properly. Crossover is excluded in order to prevent duplicates, which will cause conflicts of channel numbers and severe interference. In addition, the mutation procedure is modified and a new procedure called 'spread' is added. The following are the detailed explanations of changes and additions.

\subsection{Selection and Spread}

First, before the mutation procedure begins, the fitness score of each individual, which is the total throughput rate of the system corresponding to the current locations of UEs and channel state in the RA table, is evaluated. Then, the individuals with the highest fitness score are selected and they clone themselves to replace their adjacent individuals with their clones. The spread procedure designates the highest-scored individuals so that they become immune to the mutation procedure which follows after the spread procedure. On the other hand, clones of the highest-scored individuals will still be subject to mutation because they have a higher chance of having better fitness scores when they mutate, compared to the other randomly-generated individuals and possibly to the original highest-scored individuals.

\subsection{Mutation}

Unlike the traditional GA, we have modified and combined the procedures of mutation and crossover to prevent duplicate elements since the RA table must not include the same channel numbers within adjacent coverage areas. Duplicate elements will result in conflicts of signals on the same frequency domain and cause severe interference. Thus, rather than randomly changing the elements 
to any number, the individual will exchange its elements with the other elements of itself, preventing duplicates and still taking the advantages of mutation and crossover at the same time. The process of our proposed method is shown in Figure 2.

Episode

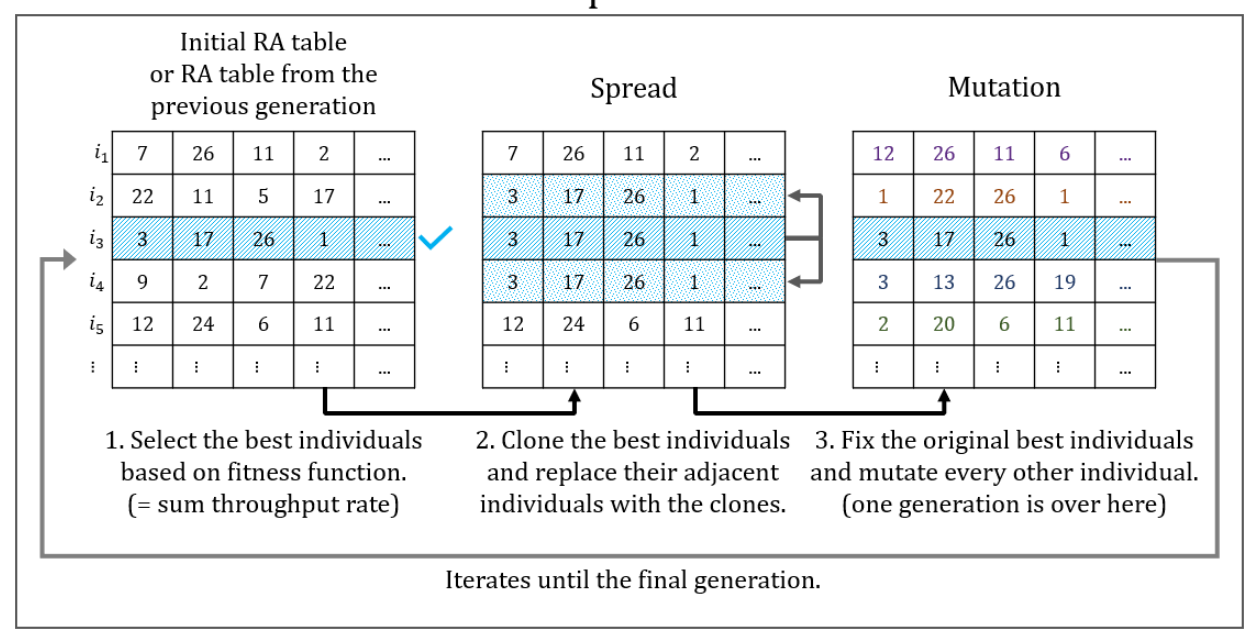

Figure 2. The evolution process within an episode. $i_{n}$ indicates each individual.

\subsection{Continuous Pool}

The process of evolution from the first generation to the last generation is called an episode. When an episode is over, UEs in the system will receive the RA table. We use the idea of continuous pool at this moment, by handing over the last population to the next episode. By doing so, the population will already be in a state where it has almost converged since the locations of UEs would have not changed in a great measure at once. Instead, the relative position of UEs will be almost the same. Thus, rather than start over the whole evolution process again, our scheme using continuous pool aims to provide a quick convergence by using the population which has converged in the last episode. The concept of continuous pool is shown in Figure 3.

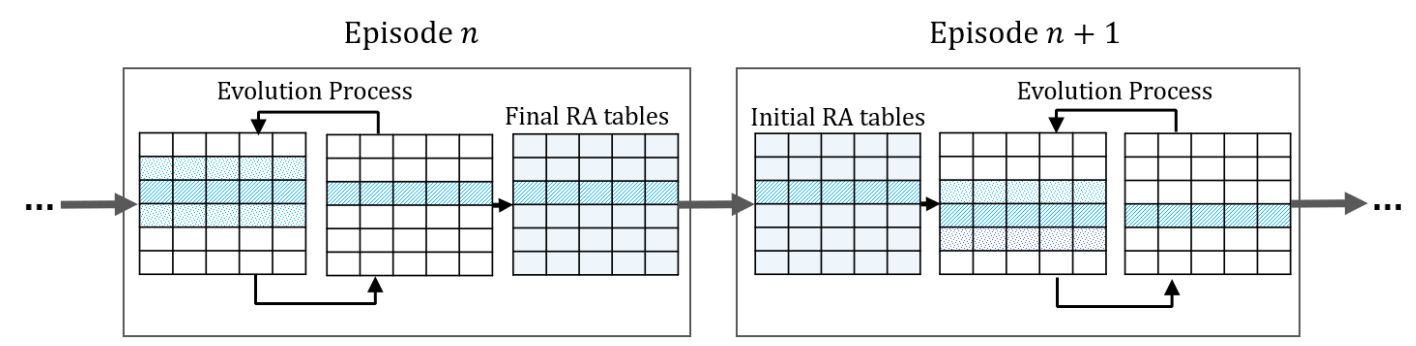

Figure 3. Continuous pool keeps the final resource allocation (RA) table from the last episode to minimize the convergence time of the current episode.

By aiming to maximize the sum throughput rate which is the sum of V2V and V2I throughput, we have observed that GA does a great job in achieving user fairness between V2V and V2I transmission. It can be explained by how GA aims to achieve a high fitness score. It does not only focus on increasing either one of V2V or V2I throughput, but both of them combined. The process of GA with continuous pool is as follows in Algorithm 1.

The algorithm first initializes the RA table and locations of every UE and BS. At this point, every individual in the RA table has randomly generated channel numbers. UEs, namely the vehicles are randomly placed on the simulated road and their $x$ - and $y$-axes are used as their location data to calculate the throughput. A total of nine BSs are placed in the simulation as described in Section 3. 
Within the episode loop, there is a generation loop. In the generation loop, the fitness scores of the individuals in the current RA table are evaluated by calculating the throughput. Then, individuals with the highest fitness scores are designated and allocated to the best_individuals list. Based on the best_individuals list, spread is performed throughout the RA table. After that, mutation is performed for each individual based on the mutation probability. However, the best individuals selected at the beginning of the current generation are not mutated in this step to prevent the decrease in fitness scores.

From the point of view considering the actual deployment, when the generation loop is over, every UE receives the RA table from the cooperative BS. The UEs will use the RA table for the rest of the 1 second until they move. In our simulation, the UEs move $1 \mathrm{~m}, 2 \mathrm{~m}$, or $3 \mathrm{~m}$, discretely. After the UEs have moved, an episode will run again to find the suitable RA table for the current channel state.

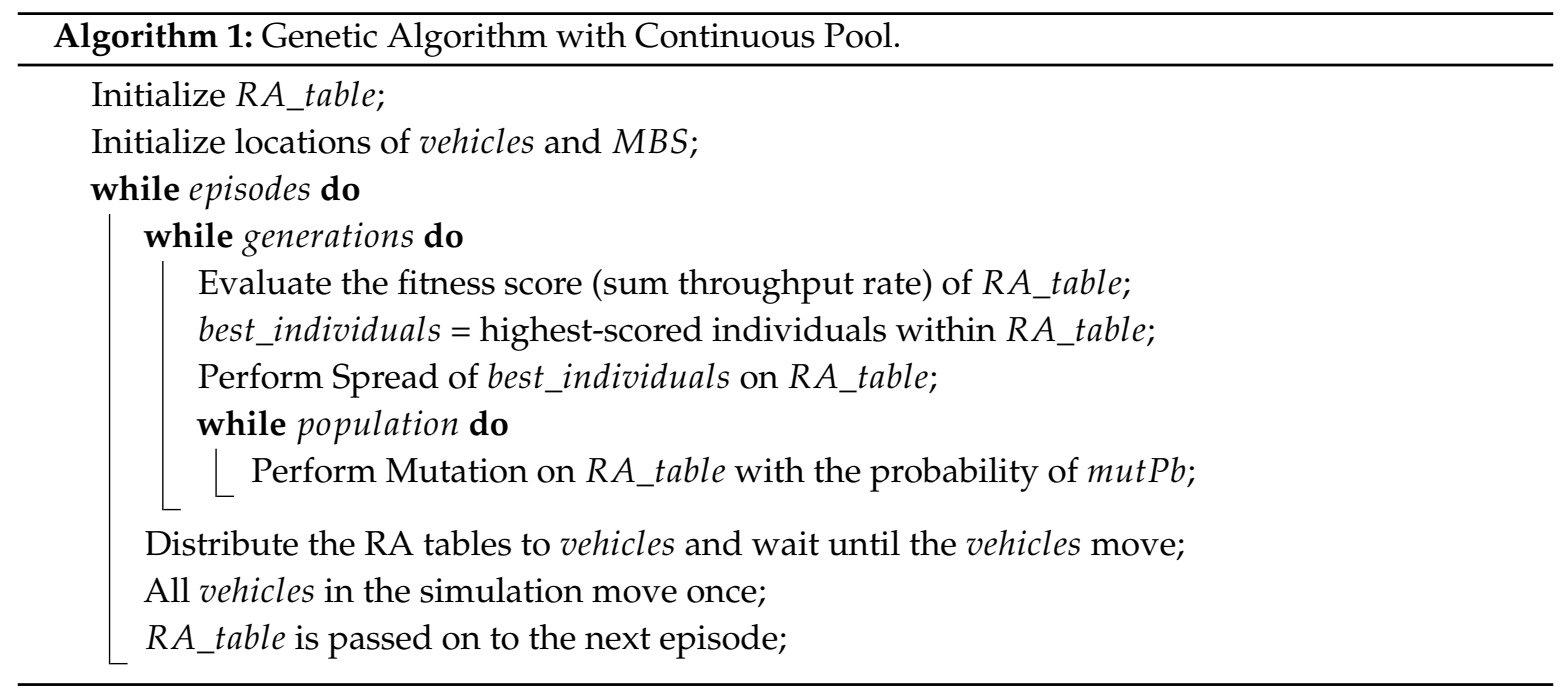

To analyze the complexity of our proposed algorithm, the number of generations, vehicles, and pool size should be considered. Firstly, in the generation loop, the fitness score of each vehicle is evaluated. Then, the two operations below take very little time that it can be ignored. In the population loop, the mutation process is carried as much as the number of pools. The whole process stated above is repeated for the number of generations. As a result, we can assess the complexity of each episode in our algorithm as follows:

$$
\text { O (generations } \times \text { vehicles } \times \text { pool size })
$$

After each episode, the RA table made with the proposed method can be broadcasted to the users through an uplink or downlink control channel in the subframe such as the physical downlink control channel (PDCCH), by the cooperative BS. Therefore, the vehicles can consistently receive the updated RA tables.

\section{Performance Evaluation}

We have evaluated the performance of the proposed scheme under a simulation that is based on our target system. In the simulation, there are a total of four roads with six lanes each and the lanes are separated by a centerline, e.g., vehicles on the half of the lanes, which is in this case, three lanes, go forward and on the other half go backward. Every road has the same length of $2100 \mathrm{~m}$ and two roads are placed in the horizontal axis and the other two are in the vertical axis with the same distance between them, forming four intersections at the position where the horizontal and the vertical roads overlap with each other. The simulation implemented based on the description above is shown in Figure 4. 


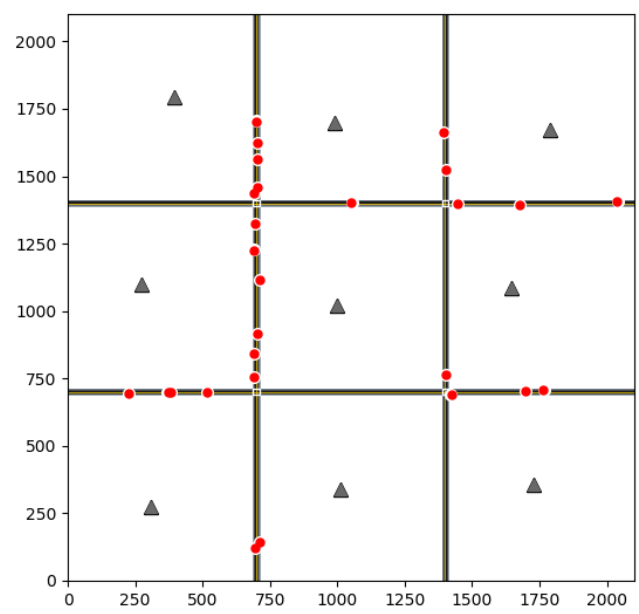

(a)

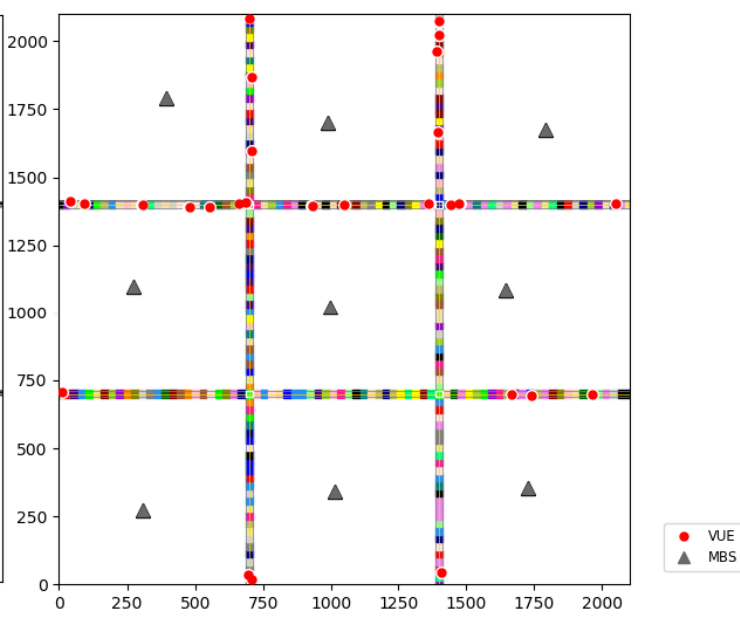

(b)

Figure 4. The simulation developed based on the target system model. Two instances of simulations (a) without hyper-fraction zones and (b) with hyper-fraction zones are shown to help comprehension of the simulation. Red circles represent vehicles and khaki triangles represent macro base stations (MBSs).

Thus, the roads will also form square-shaped blocks between each other and we assume there are many buildings within the block, and an MBS is located on the rooftop of a building that is near the center of the block. Therefore, we assume an urban area where the signals will deteriorate as they travel through the environment, so we apply the log distance path loss model in our simulation.

The simulation is conducted under the condition shown in Table 1. Our simulation parameters and communication requirements are based on cellular vehicle-to-everything (C-V2X) communication referring to [34]. We have observed a significant increment in total throughput rate using GA with continuous pool compared to conventional GA and the random search method.

Table 1. Simulation Parameters.

\begin{tabular}{cc}
\hline Parameters & Values \\
\hline Mobility model & Manhattan mobility model \\
Channel model & Log distance path-loss model \\
Types of communications & V2V, V2I NOMA \\
Transmit power from UE & $23 \mathrm{dBm}$ \\
\# of vehicles & $9,18,27,36,45$ \\
\# of channels & 9 \\
\# of macro base stations & 9 \\
\# of hyper-fraction zones & 304 \\
\# of episodes & 10 \\
Distance vehicles move per episode & $1 \mathrm{~m}, 2 \mathrm{~m}, 3 \mathrm{~m}$ \\
\# of generations per episode & 1000 \\
Size of pool & 30 individuals $(\mathrm{RA}$ tables $)$ \\
Size of simulation environment & $2100 \times 2100 \mathrm{~m}^{2}$ \\
Size of hyper-fraction zone & $28 \times 28 \mathrm{~m}^{2}$ \\
$\zeta$ for shadowing of V2V and V2I & $4 \mathrm{~dB}, 8 \mathrm{~dB}[35]$ \\
\hline
\end{tabular}

In Figure 5, growth of the average V2V and V2I NOMA throughput rate from each episode is shown. It can be observed that the throughput rates between the proposed method and the conventional method already differ from the beginning due to the advantages of using unique evolution procedures in the proposed method. Moreover, as the episode continues and cars keep moving, we can see that the throughput rate of the proposed method is almost always superior to the conventional method within the given number of generations. This is because our proposed scheme keeps the RA tables of the last episode, whereas the conventional method does not. In addition, in the later stage of 
the episodes, it can be observed that the throughput rate of the proposed method does not increase very much because it is already in a state where it has almost converged to the most optimal solution possible for GA through many generations of the evolution process, which can verify the advantage of using continuous pool.
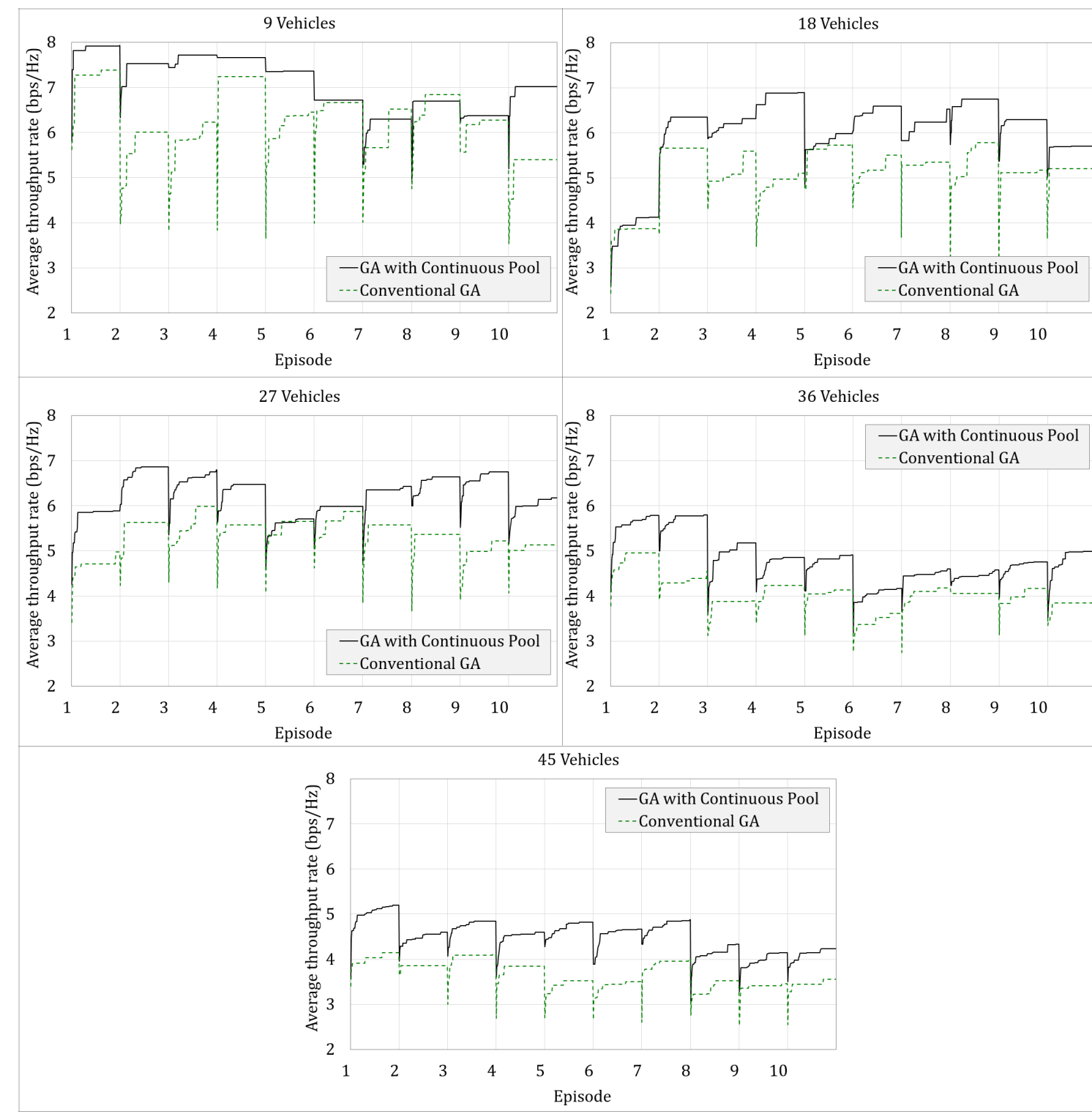

Figure 5. Average of vehicle-to-vehicle-vehicle-to-infrastructure non-orthogonal multiple accesscan (V2V-V2I NOMA) throughput rate link using hyper-fraction with genetic algorithm (GA) with continuous pool and without (conventional GA).

In Figure 6, a comparison of the average of V2V and V2I NOMA throughput rate of 10 episodes using each proposed method, conventional GA, and random search method is shown. It is shown that the proposed method performs approximately $15 \%$ better than the conventional GA and $65 \%$ better than the random search method under the same simulation settings. 


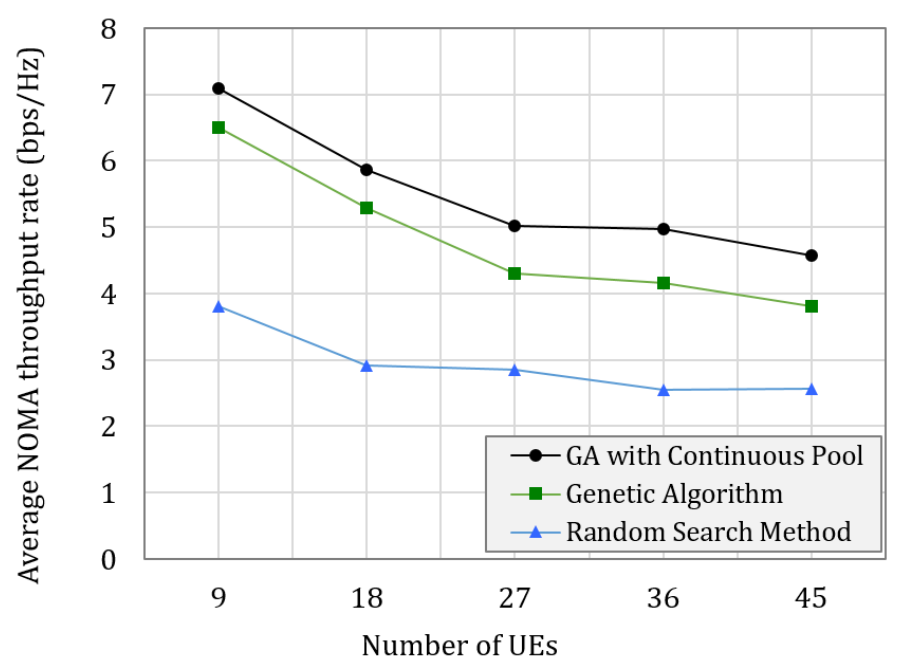

Figure 6. Comparison of average throughput among GA with continuous pool, conventional GA, and random search method.

In Figure 7, the average values of throughput rates from six different sizes of pool are shown. As mentioned earlier, a pool is a group consisting of individuals and the size of a pool is the number of RA tables in a pool.

Having more individuals in a pool resulted in a slightly better throughput of the system because the evolution process benefits from more options and possibilities of exploration for better solutions. In exchange, the computational complexity increases as the size of pool increases. The simulation is conducted with 45 vehicles, 1000 generations per each episode, and 10 episodes in total. The throughput rate shown on the graph is the average value of NOMA link throughput rates in the last generation in each of all 10 episodes.

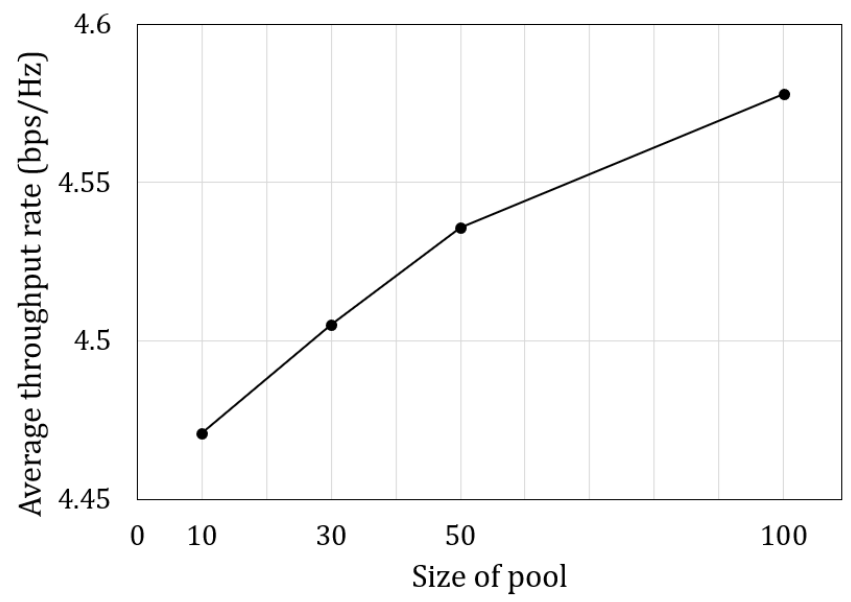

Figure 7. Average throughput according to the size of pool. Results show average values of 10 episodes.

In Figure 8, average throughput rate per NOMA link in each episode according to the size of pool is shown. In this result, GA with continuous pool is used for four different simulation instances, and conducted with 45 vehicles in the system. The only difference among those instances is the size of pool. It can be observed that larger sizes of pool tend to have slightly greater average throughput rate. However, since the method is based on a heuristic algorithm, in some episodes, it is shown that better results with a larger pool size are not always guaranteed. 


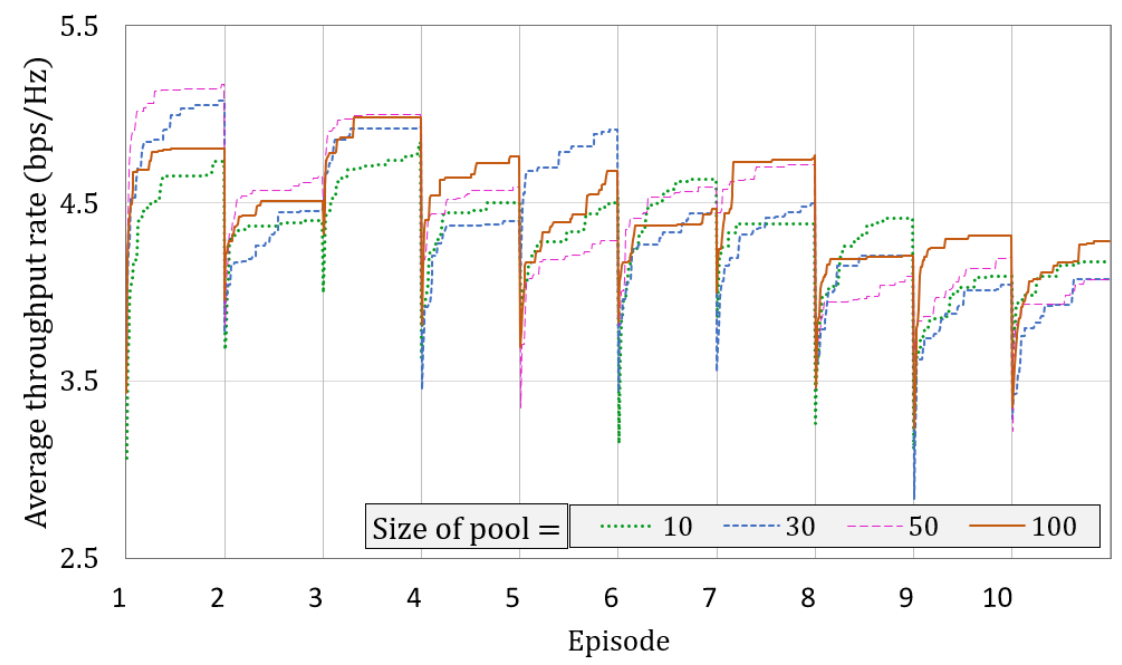

Figure 8. Comparison of average throughput in each episode according to the size of pool.

\section{Discussion}

In this paper, we proposed the concepts of hyper-fraction and GA with continuous pool to address the resource allocation problem in NOMA V2V and V2I uplink systems by improving the latency and throughput rate. We demonstrated in the results section how efficient the GA with continuous pool is compared to conventional methods in continuous situations such as vehicular networks. In addition, we proposed the idea of hyper-fraction and discussed its advantages over FFR. We will continue our research on NOMA vehicular networks and find more efficient ways in respect of throughput rate, user fairness, and latency.

Author Contributions: Conceptualization, methodology, software, formal analysis, S.L. and J.K.; validation, J.P. and S.C.; investigation, data curation, writing - original draft preparation, visualization, S.L; writing - review and editing, J.P.; supervision, project administration, and funding acquisition, S.C. All authors have read and agreed to the published version of the manuscript.

Funding: This work was supported by Institute for Information \& communications Technology Promotion(IITP) grant funded by the Korea government(MSIT) (No. 2018-0-00969, Full duplex non-orthogonal multiple access (NOMA) optimization technologies using deep learning for 5G based autonomous vehicular networks) and was supported by the research fund of Hanyang University (HY-2015-N).

Conflicts of Interest: The authors declare no conflict of interest.

\section{Abbreviations}

The following abbreviations are used in this manuscript:

$\begin{array}{ll}\text { BS } & \text { Base Station } \\ \text { CSI } & \text { Channel State Information } \\ \text { C-V2X } & \text { Cellular Vehicle-to-Everything } \\ \text { D2D } & \text { Device-to-Device } \\ \text { MBS } & \text { Macro Base Station } \\ \text { FFR } & \text { Fractional Frequency Reuse } \\ \text { GA } & \text { Genetic Algorithm } \\ \text { HF-zone } & \text { Hyper-Fraction Zone } \\ \text { NOMA } & \text { Non-Orthogonal Multiple Access } \\ \text { OMA } & \text { Orthogonal Multiple Access } \\ \text { OFDMA } & \text { Orthogonal Frequency-Division Multiple Access } \\ \text { RA } & \text { Resource Allocation } \\ \text { SC-FDMA } & \text { Single-Carrier Frequency Division Multiple Access } \\ \text { SIC } & \text { Successive Interference Cancellation }\end{array}$


SINR Signal-to-Interference-plus-Noise Ratio

UE User Equipment

V2I Vehicle-to-Infrastructure

V2V Vehicle-to-Vehicle

\section{References}

1. Ge, X.; Tu, S.; Mao, G.; Wang, C.; Han, T. 5G Ultra-Dense Cellular Networks. IEEE Wirel. Commun. 2016, 23, 72-79. [CrossRef]

2. Agiwal, M.; Roy, A.; Saxena, N. Next, Generation 5G Wireless Networks: A Comprehensive Survey. IEEE Commun. Surv. Tutor. 2016, 18, 1617-1655. [CrossRef]

3. Fan, P.; Zhao, J.; Chih-Lin, I. 5G high mobility wireless communications: Challenges and solutions. China Commun. 2016, 13,1-13. [CrossRef]

4. Shah, S.A.A.; Ahmed, E.; Imran, M.; Zeadally, S. 5G for Vehicular Communications. IEEE Commun. Mag. 2018, 56, 111-117. [CrossRef]

5. Guo, S.; Zhou, X. Robust Resource Allocation With Imperfect Channel Estimation in NOMA-Based Heterogeneous Vehicular Networks. IEEE Trans. Commun. 2019, 67, 2321-2332. [CrossRef]

6. MacHardy, Z.; Khan, A.; Obana, K.; Iwashina, S. V2X Access Technologies: Regulation, Research, and Remaining Challenges. IEEE Commun. Surv. Tutor. 2018, 20, 1858-1877. [CrossRef]

7. Husain, S.S.; Kunz, A.; Prasad, A.; Pateromichelakis, E.; Samdanis, K. Ultra-High Reliable 5G V2X Communications. IEEE Commun. Stand. Mag. 2019, 3, 46-52. [CrossRef]

8. Di, B.; Song, L.; Li, Y.; Han, Z. V2X Meets NOMA: Non-Orthogonal Multiple Access for 5G-Enabled Vehicular Networks. IEEE Wirel. Commun. 2017, 24, 14-21. [CrossRef]

9. Dai, L.; Wang, B.; Ding, Z.; Wang, Z.; Chen, S.; Hanzo, L. A Survey of Non-Orthogonal Multiple Access for 5G. IEEE Commun. Surv. Tutor. 2018, 20, 2294-2323. [CrossRef]

10. Liu, Y.; Qin, Z.; Elkashlan, M.; Ding, Z.; Nallanathan, A.; Hanzo, L. Non-orthogonal multiple access for 5G and beyond. arXiv 2018, arXiv:1808.00277.

11. Saito, Y.; Kishiyama, Y.; Benjebbour, A.; Nakamura, T.; Li, A.; Higuchi, K. Non-Orthogonal Multiple Access (NOMA) for Cellular Future Radio Access. In Proceedings of the 2013 IEEE 77th Vehicular Technology Conference (VTC Spring), Dresden, DE, USA, 2-5 June 2013; pp. 1-5.

12. Ding, Z.; Liu, Y.; Choi, J.; Sun, Q.; Elkashlan, M.; Chih-Lin, I.; Poor, H.V. Application of Non-Orthogonal Multiple Access in LTE and 5G Networks. IEEE Commun. Mag. 2017, 55, 185-191. [CrossRef]

13. Shin, W.; Vaezi, M.; Lee, B.; Love, D.J.; Lee, J.; Poor, H.V. Non-Orthogonal Multiple Access in Multi-Cell Networks: Theory, Performance, and Practical Challenges. IEEE Commun. Mag. 2017, 55, 176-183. [CrossRef]

14. Ding, Z.; Lei, X.; Karagiannidis, G.K.; Schober, R.; Yuan, J.; Bhargava, V.K. A Survey on Non-Orthogonal Multiple Access for 5G Networks: Research Challenges and Future Trends. IEEE J. Sel. Areas Commun. 2017, 35, 2181-2195. [CrossRef]

15. Mostafa, A.E.; Zhou, Y.; Wong, V.W.S. Connectivity maximization for narrowband IoT systems with NOMA. In Proceedings of the 2017 IEEE International Conference on Communications (ICC), Paris, France, 21-25 May 2017; pp. 1-6.

16. Chen, Y.; Wang, L.; Ai, Y.; Jiao, B.; Hanzo, L. Performance Analysis of NOMA-SM in Vehicle-to-Vehicle Massive MIMO Channels. IEEE J. Sel. Areas Commun. 2017, 35, 2653-2666. [CrossRef]

17. Ahlem, M.; Mnif, K.; Zarai, F. A Survey on Radio Resource Allocation for V2X Communication. Wirel. Commun. Mob. Comput. 2019, 4, 1-12. [CrossRef]

18. Budhiraja, I.; Tyagi, S.; Tanwar, S.; Kumar, N.; Guizani, M. CR-NOMA Based Interference Mitigation Scheme for $5 \mathrm{G}$ Femtocells Users. In Proceedings of the 2018 IEEE Global Communications Conference (GLOBECOM), Abu Dhabi, UAE, 9-13 December 2018; pp. 1-6.

19. Di, B.; Song, L.; Li, Y.; Li, G.Y. NOMA-Based Low-Latency and High-Reliable Broadcast Communications for 5G V2X Services. In Proceedings of the GLOBECOM 2017-2017 IEEE Global Communications Conference, Singapore, 4-8 December 2017; pp. 1-6.

20. Chen, S.; Hu, J.; Shi, Y.; Peng, Y.; Fang, J.; Zhao, R.; Zhao, L. Vehicle-to-Everything (v2x) Services Supported by LTE-Based Systems and 5G. IEEE Commun. Stand. Mag. 2017, 1, 70-76. [CrossRef] 
21. Altay, C.; Koca, M. Fractional Frequency Reuse in Non-Orthogonal Multiple Access Heterogeneous Networks. In Proceedings of the 2018 IEEE International Conference on Communications (ICC), Kansas City, MO, USA, 20-24 May 2018; pp. 1-6.

22. Gemici, Ö.F.; Kara, F.; Hokelek, I.; Kurt, G.K.; Çırpan, H.A. Resource allocation for NOMA downlink systems: Genetic algorithm approach. In Proceedings of the 2017 40th International Conference on Telecommunications and Signal Processing (TSP), Barcelona, Spain, 5-7 July 2017; pp. 114-118.

23. Saito, Y.; Benjebbour, A.; Kishiyama, Y.; Nakamura, T. System-level performance evaluation of downlink non-orthogonal multiple access (NOMA). In Proceedings of the 2013 IEEE 24th Annual International Symposium on Personal, Indoor, and Mobile Radio Communications (PIMRC), London, UK, 8-11 September 2013; pp. 611-615.

24. Zhang, N.; Wang, J.; Kang, G.; Liu, Y. Uplink Nonorthogonal Multiple Access in 5G Systems. IEEE Commun. Lett. 2016, 20, 458-461. [CrossRef]

25. abassum, H.; Ali, M.S.; Hossain, E.; Hossain, M.; Kim, I. Non-Orthogonal Multiple Access (NOMA) in Cellular Uplink and Downlink: Challenges and Enabling Techniques. arXiv 2016, arXiv:1608.05783.

26. Ali, M.S.; Tabassum, H.; Hossain, E. Dynamic User Clustering and Power Allocation for Uplink and Downlink Non-Orthogonal Multiple Access (NOMA) Systems. IEEE Access 2016, 4, 6325-6343. [CrossRef]

27. Di, B.; Song, L.; Li, Y.; Li, G.Y. Non-Orthogonal Multiple Access for High-Reliable and Low-Latency V2X Communications in 5G Systems. IEEE J. Sel. Areas Commun. 2017, 35, 2383-2397. [CrossRef]

28. Zhang, D.; Liu, Y.; Dai, L.; Bashir, A.K.; Nallanathan, A.; Shim, B. Performance Analysis of FD-NOMA-Based Decentralized V2X Systems. IEEE Trans. Commun. 2019, 67, 5024-5036. [CrossRef]

29. Guo, S.; Zhou, X. Robust power allocation for NOMA in heterogeneous vehicular communications with imperfect channel estimation. In Proceedings of the 2017 IEEE 28th Annual International Symposium on Personal, Indoor, and Mobile Radio Communications (PIMRC), Montreal, QC, Canada, 8-13 October 2017; pp. 1-5.

30. Sheng, Z.; Su, X.; Zhang, X. A Novel Power Allocation Method for Non-orthogonal Multiple Access in Cellular Uplink Network. In Proceedings of the 2017 International Conference on Intelligent Environments (IE), Seoul, Korea, 21-25 August 2017; pp. 157-159.

31. He, J.; Tang, Z.; Ding, Z.; Wu, D. Successive Interference Cancellation and Fractional Frequency Reuse for LTE Uplink Communications. IEEE Trans. Veh. Technol. 2018, 67, 10528-10542. [CrossRef]

32. Kim, J.; Kim, T.; Noh, J.; Cho, S. Fractional Frequency Reuse Scheme for Device to Device Communication Underlaying Cellular on Wireless Multimedia Sensor Networks. Sensors 2018, 18, 2661. [CrossRef] [PubMed]

33. Ali, M.S.; Hossain, E.; Kim, D.I. Non-orthogonal multiple access (NOMA) for downlink multiuser mimo systems: User clustering, beamforming, and power allocation. IEEE Access 2016, 5, 565-577. [CrossRef]

34. Sabeeh, S.; Sroka, P.; Wesołowski, K. Estimation and Reservation for Autonomous Resource Selection in C-V2X Mode 4. In Proceedings of the 2019 IEEE 30th Annual International Symposium on Personal, Indoor and Mobile Radio Communications (PIMRC), Istanbul, Turkey, 8-11 September 2019; pp. 1-6. [CrossRef]

35. 3rd Generation Partnership Project: Technical Specification Group Radio Access Network: Study on LTE-Based V2X Services: (Release 14), Standard 3GPP TR 36.885 V2.0.0, June 2016. Available online: https:/ / portal.3gpp.org/desktopmodules/Specifications/SpecificationDetails.aspx?specificationId= 2934 (accessed on 21 June 2020).

(C) 2020 by the authors. Licensee MDPI, Basel, Switzerland. This article is an open access article distributed under the terms and conditions of the Creative Commons Attribution (CC BY) license (http:/ / creativecommons.org/licenses/by/4.0/). 